\title{
REPRESENTATION OF ORDERED LINEAR SPACES
}

\author{
RICHARD C. METZLER
}

\begin{abstract}
An approach to the theory of representation of ordered linear spaces by functions is presented which presupposes only linear algebra and general topology. The same method is shown to apply to both the order unit case and the linear lattice case; previously these have been treated separately. Also some applications to the elementary theory of Choquet boundaries are presented and the Krein-Milman theorem is derived.
\end{abstract}

A familiar technique in modern mathematics is the representation of an abstract, axiomatically defined mathematical concept as a concrete mathematical object. A student usually encounters this technique for the first time when he learns that an $n$-dimensional linear space (i.e., vector space) is isomorphic to $R^{n}$. Representations in many cases allow mathematicians to check conjectures and prove theorems much more easily than in the abstract situation. In the case of ordered linear spaces and linear lattices in particular there have been many methods presented for representation of these spaces as function spaces $([4],[5],[7],[10],[11])$. A detailed treatment of these methods and others can be found in the book of Luxembourg and Zaanen, [6].

In general these methods require considerable knowledge of the theory of ordered linear spaces. Also the approach used for spaces with an order unit is quite different from that used for linear lattices. In this article we present an approach to representation theory which presupposes only linear algebra and general topology and which works in both the order unit case and the linear lattice case. We also present some applications to the elementary theory of Choquet boundaries and derive the Krein-Milman theorem.

1. Extremal Monotonic Linear Functionals. Let $V$ be a linear space over the reals. A reflexive and transitive (but not necessarily antisymmetric) relation "ฏ” is said to be a linear ordering of $V$ if $x \leqq y$ implies $x+z \leqq y+z$ for all $z$ in $V$, and $x \leqq y$ implies $r x \leqq r y$ for all nonnegative real numbers $r$. With a linear ordering, $V$ is said to be an ordered linear space. The set $V^{+}=\{x \in V: x \geqq 0\}$ forms a wedge

Received by the editors October 1, 1973.

Copyright $\odot 1975$ Rocky Mountain Mathematics Consortium 
in $V$ (i.e., $V^{+}+V^{+} \subset V^{+}$and $r V^{+} \subset V^{+}$for $r \geqq 0$ ) and we have $x \leqq y$ if and only if $y-x$ is in $V^{+}$. The ordering is antisymmetric if and only if $V^{+} \cap\left(-V^{+}\right)=\{0\}$. A subset $S$ of $V$ is said to be upward directed if, for any two elements of $S$, there is a third element of $S$ larger than both. In the following we will designate the real numbers by $R$.

A linear function $f$ from $V$ to another ordered linear space $Y$ is said to be monotonic if $x \leqq y$ in $V$ implies $f(x) \leqq f(y)$ in $Y$. If $f$ is a monotonic linear functional defined on a subspace $G$ of $V$ we define a new function $\underline{f}$ by $\underline{f}(x)=\sup f\left(x-V^{+}\right)$(i.e., $\underline{f}(x)=\sup \{f(y)$ : $x \geqq y \in G\}$ ) for all $x$ in $G+V^{+}$such that the sup exists in $R$. We define the dual function $\bar{f}$ by $\bar{f}(x)=\inf f\left(x+V^{+}\right)$for all $x$ in $G-V^{+}$such that the inf exists and note the obvious fact that $\bar{f}(x)=-\underline{f}(-x)$ for all $x$ in $\operatorname{dmn} \bar{f}$. It is easy to see that the set of monotonic linear functionals forms a wedge in the algebraic dual of $V$. If we define $f \leqq g$ in the algebraic dual to mean that $g-f$ is monotonic then the algebraic dual becomes an ordered linear space. (This would not be true if we had required antisymmetry in our definition of ordered linear space. The dual space is antisymmetric if and only if $V$ is upward directed.)

An element $x$ in $V^{+}$is said to be an extremal point of $V^{+}$if, for each $y$ in $V$ such that $0 \leqq y \leqq x$, we have $y=r x$ for some real number $r$ such that $0 \leqq r \leqq 1$. To give a geometrical feel for this notion we point out that in 3-space the extremal elements of a right circular cone are all the elements on the surface of the cone; if we slice this cone in half with a plane through the axis to get a cone with semicircular cross section then the elements on the flat surface are not extremal. In the "positive octant" cone in 3-space the extremal elements are those elements on the positive $x, y$, and $z$ axes.

A monotonic linear functional defined on a subspace $G$ of $V$ is said to be extremal if it is an extremal point of the wedge of monotonic linear functionals on $G$ considered as a subset of the ordered vector space of all linear functionals on $G$.

A simple example of an ordered linear space is provided by the space of all real-valued functions defined on some set with the usual pointwise definitions of the linear operation and the order. Problem: To what extent can all ordered linear spaces be considered as subspaces of such a function space? If we take any subset $A$ of the algegraic dual of $V$ we can map each $x$ in $V$ to a function $\hat{x}$ on $A$ by defining $\hat{x}(f)=f(x)$ for all $f$ in $A$. Now if the set $A$ is too small (e.g., let $A$ be a one-element set) the mapping $x \rightarrow \hat{x}$ is not one-to-one in general. On the other hand we do not want $A$ to be too large; we 
want the representation to be such that positive elements go to positive functions and such that any finite sups which exist are preserved as pointwise sups. The successful approach turns out to be that of choosing the functionals in $A$ from the extremal functionals on $V$. In order to show that there exist enough of the kind of functionals we want we show how to obtain them by extension from very simple functionals. We first need a "one more dimension" extension theorem which combines results found in [3, pp. 24-33].

1.1 THEOREM. Let $f$ be a monotonic linear functional defined on a subspace $G$ of an ordered linear space $V$. For $x$ in $\operatorname{dmn} \underline{f}$ suppose $f_{1}$ to be defined on $G+R x$ by $f_{1}(y+r x)=f(y)+r \underline{f}(x)$. Then:

(a) $f_{1}$ is a monotonic linear function;

(b) if $f$ is extremal then $f_{1}$ is extremal;

(c) if $f_{1}$ is extremal and if the set $\{y \in G: y \leqq x\}$ is upward directed, then $f$ is extremal.

REMARK. Of course a dual result holds for $x \operatorname{in} \operatorname{dmn} \bar{f}$ as can be seen by multiplying by minus one.

Proof. If $x$ is in $G$ it is immediate that $f_{1}=f$ while if $x$ is not an element of $G$ then it is easy to see that the function $f_{1}$ is well-defined and linear. To see that it is monotonic it is enough to show that $0 \leqq y+r x$ implies $0 \leqq f_{1}(y+r x)$. If $r \geqq 0$ we have $-y \leqq r x$ implies $f(-y) \leqq \underline{f}(r x)=r \underline{f}(x)$ so we conclude that $0 \leqq f_{1}(y+r x)$. If $r<0$ then $-(1 / r) y \geqq x$ implies $f(-(1 / r) y) \geqq \underline{f}(x)$ and we again obtain $0 \leqq f_{1}(y+r x)$. Hence statement (a) is proved.

To prove statement (b) suppose that $f$ is extremal and let $g$ be a linear functional defined on $G+R x$ such that $0 \leqq g \leqq f_{1}$. By extremality of $f$ there exists $r$ between 0 and 1 such that, for every $y$ in $G, g(y)=r f(y)$. We need only show that $g(x)=r \underline{f}(x)$ and this results from the following inequalities:

$$
\begin{gathered}
g(x) \geqq(\underline{r f})(x)=\underline{r} \underline{f}(x)=r f_{1}(x), \\
\left(f_{1}-g\right)(x) \geqq\left(\underline{f-g)}(x)=(1-r) \underline{f}(x)=(1-r) f_{1}(x) .\right.
\end{gathered}
$$

To prove statement (c) suppose that $f_{1}$ is extremal and let $g$ be a linear functional defined on $G$ such that $0 \leqq g \leqq f$. Then

$f_{1}(x)=\underline{f}(x)=\sup \{(g+(f-g))(y): x \geqq y \in G\}=\underline{g}(x)+(\underline{f-g})(x)$

where the last equality is a consequence of the fact that $\{y \in G: y \leqq x\}$ is upward directed.

Hence if we define $g_{1}$ and $(f-g)_{1}$ on $G+R x$ so that $g_{1}(x)=g(x)$ and $(f-g)_{1}(x)=(f-g)(x)$ then $f_{1}=g_{1}+(f-g)_{1}$. But from 
part (a) we see that $g_{1}$ and $(f-g)_{1}$ are monotonic linear mappings so we conclude that $0 \leqq g_{1} \leqq f_{1}$. Since $f_{1}$ is extremal we have $g_{1}=r f_{1}$ which gives $g=r f$. Hence $f$ is extremal.

Given an ordered linear space $V$ we define $\mathcal{M}(V)$ (resp. $\mathcal{E}(V))$ to be the collection of all monotonic (resp. extremal) linear functionals with domains contained in $V$, ordered by inclusion as sets of ordered pairs. We designate by $M(V)$ (resp. $E(V)$ ) the set of maximal elements of $\mathcal{M}(V)$ (resp. $\mathcal{E}(V))$.

1.2 TheOREM. Let $f$ be a monotonic linear functional defined on a subspace of an ordered linear space $V$. There exists an extension of $f$ which is in $M(V)$ and if $f$ is extremal there exists an extension of $f$ which is in $E(V)$. Furthermore in either case if $f_{M}$ is such an extension we have:

$$
\begin{aligned}
& \operatorname{dmn} f_{M}= \\
& \left(\operatorname{dmn} f_{M}-V^{+}\right) \cap\left(\operatorname{dmn} f_{M}+V^{+}\right) \supset\left(\operatorname{dmn} f-V^{+}\right) \cap\left(\operatorname{dmn} f+V^{+}\right) .
\end{aligned}
$$

Proof. It is routine to verify that $\mathcal{M}(V)$ (resp. $\mathcal{E}(V)$ ) satisfies the hypotheses of Zorn's lemma. Thus a maximal extension $f_{M}$, of the required sort, does exist and since $x \in\left(\operatorname{dmn} f_{M}-V^{+}\right) \cap\left(\operatorname{dmn} f_{M}\right.$ $+V^{+}$) implies $x \in \operatorname{dmn} f_{M}$ we see that $f_{M}$ must be defined on $x$ by the previous theorem and maximality. The last inclusion is immediate.

2. Representation of Order Unit Spaces. An element $u$ of an ordered linear space $V$ is said to be an order unit if, for each $x$ in $V$, there is a positive real number $r$ such that $-r u \leqq x \leqq r u$. We say that a pair $(V, u)$ is an order unit space if $u$ is an order unit for $V$ which is nonnegative. (The last requirement eliminates the uninteresting case when $V=V^{+}$.) Given an order unit space $(V, u)$ we define a function $U: R u \rightarrow R$ by $U(r u)=r$. Also we define $E(V, u)$ to be the subset of $E(V)$ composed of functionals which take the value one on $u$. From theorem 1.2 we see that $\operatorname{dmn} f=V$ for all $f$ in $E(V, u)$.

2.1 Theorem. Let $(V, u)$ be an order unit space. Then, for each $x$ in $V$, there exists $f_{1}$ and $f_{2}$ in $E(V, u)$ such that $f_{1}(x)=\underline{U}(x)$ and $f_{2}(x)=\bar{U}(x)$.

Proof. The requirement that $u$ be nonnegative ensures that $U$ is extremal and that $\operatorname{dmn} \underline{U}=\operatorname{dmn} \bar{U}=V$. By 1.1 , for any $x$ in $V$, we can extend $U$ to an extremal functional $U_{1}$ defined on $R u+$ $R x$ in such a way that $U_{1}(x)=\underline{U}(x)$. Then any extremal extension of $U_{1}$ to the whole space (possible by 1.2 and the fact that $u$ is an order 
unit) serves as the required $f_{1}$. The result for $\bar{U}(x)$ follows by consideration of $-x$.

Since all functionals in $E(V, u)$ are defined on all of $V$, given any $x$ in $V$ we may define $\hat{x}$ in $R^{E(V, u)}$ by $\hat{x}(f)=f(x)$ for all $f$ in $E(V, u)$. It is routine to verify that the mapping $x \rightarrow \hat{x}$ is a monotonic linear function from $V$ to a subspace of $R^{E(V, u)}$ (where the linear operations and the order are defined pointwise as usual.) We now investigate conditions which will ensure: (a) that the mapping is one-to-one; or (b) that any element with a positive image is positive.

An ordered linear space $V$ is said to be almost Archimedean if, for any pair $\{x, y\}$ in $V$ such that $-y \leqq n x \leqq y$ for all $n=1,2, \cdots$, we have $x=0$. The space is said to be Archimedean if, for any pair $\{x, y\}$ in $V$ such that $n x \leqq y$ for all $n=1,2, \cdots$, we have $x \leqq 0$. It is easy to see that a space which is both Archimedean and antisymmetric is almost Archimedean.

2.2 Theorem. Let $(V, u)$ be an order unit space. The mapping $x \rightarrow \hat{x}$ of $V$ into $R^{E(V, u)}$ is one-to-one if and only if $V$ is almost Archimedean. The mapping has the property that any element with a positive image is positive if and only if $\mathrm{V}$ is Archimedean.

Proof. For any $f$ in $E(V, u)$ and $x$ in $V$ it is clear by monotonicity that $\underline{U}(x) \leqq f(x) \leqq \bar{U}(x)$. Then the previous theorem shows that we will have $\hat{x}=0$ if and only if $\underline{U}(x)=\bar{U}(x)=0$. But if $V$ is almost Archimedean then this implies that $x=0$. Conversely, if $-y \leqq n x$ $\leqq y$ for all $n=1,2, \cdots$, choose a positive real number $r$ such that $-r u \leqq y \leqq r u$. Then $-r u \leqq n x \leqq r u$ for all $n=1,2, \cdots$ implies that $\underline{U}(x)=\bar{U}(x)=0$ so the assumption that the mapping is one-toone implies $x=0$.

If $V$ is Archimedean and $x \in V$ is such that $\hat{x} \geqq 0$ then $\underline{U}(x) \geqq 0$. This implies that $-n x \leqq u$ for all $n=1,2, \cdots$ and we conclude that $x \geqq 0$. Conversely, if any element with a positive image is positive, suppose that $n x \leqq y$ for all $n=1,2, \cdots$. Then $n \hat{x}(f) \leqq \hat{y}(f)$ for all $f \in E(V, u)$ implies $\hat{x} \leqq 0$ and consequently $x \leqq 0$.

In order to represent a space $V$ which lacks an order unit the first idea that comes to mind is to add a unit to $V$-i.e., find a space $V_{1}$ with a unit $u$ such that there exists an isomorphism $B$ of $V$ into $V_{1}$. If such a $V_{1}$ exists we can use the linear functional $U$ on $R u$ to define a functional $P$ on $V$ by: $P(x)=\max \{\bar{U}(B(x)), 0\}$ for all $x$ in $V$. It is easy to verify that $P$ has the following properties:

(P1) $x \leqq y$ implies $P(x) \leqq P(y)$ for all $x$ and $y$ in $V$,

(P2) $0 \leqq P(x)$ for all $x$ in $V$, 
(P3) $P(r x)=r P(x)$ for all $x$ in $V$ and $r \geqq 0$,

(P4) $P(x+y) \leqq P(x)+P(y)$ for all $x$ and $y$ in $V$.

Conversely the next theorem shows that existence of such a functional is a sufficient condition for the existence of the desired containing order unit space.

2.3 Theorem. Given an ordered linear space $V$ with a functional $P$ defined on $V$ which satisfies $(\mathrm{P} 1)-(\mathrm{P} 4)$ above, there exists an order unit space $\left(V_{P}, u\right)$, and a linear and order isomorphism $B$ from $V$ to a subspace of $V_{P}$, such that $P(x)=\max \{\bar{U}(B(x)), 0\}=\bar{U}(B(x))$ for all $x$ in $V$.

Proof. Let $V_{P}=R \times V$ and define $V_{P}{ }^{+}=\left(\{0\} \times V^{+}\right) \cup\{(r, x)$ : $r>P(-x)\}$. First we will show that $V_{P}{ }^{+}$is a wedge. If $x \in V^{+}$and $(r, y)$ is in $V_{P}{ }^{+}$for $r>0$ then $P(-y-x) \leqq P(-y)<r$ (by monotonicity of $P$ ) so we conclude $(0, x)+(r, y) \in V_{P}{ }^{+}$. If $(s, z)$ is any other member of $V_{P}{ }^{+}$we have $P(-y-z) \leqq P(-y)+P(-z) \leqq r+s$ so $(r, y)+(s, z)$ is also in $V_{P}{ }^{+}$. The remaining details of the verification that $V_{P}{ }^{+}$is a wedge are routine.

Now if we define $(r, y) \leqq(s, z)$ in $V_{P}$ to mean that their difference is in $V_{P}{ }^{+}$it is easy to see that this makes $V_{P}$ into an ordered linear space. Furthermore the mapping $B: V \rightarrow V_{P}$ defined by $B(x)=(0, x)$ is clearly an order isomorphism of $V$ onto the subspace $\{0\} \times V$. Given any $(r, x) \in V_{P}$ let $s>r+P(x)$. Then $s-r>P(x)$ implies $s(1,0) \geqq(r, x)$. Similarly $s+r>P(-x)$ implies $(r, x) \geqq-s(1,0)$. Thus $u=(1,0)$ is an order unit for $V_{P}$ and $\bar{U}(r, x) \leqq r+P(x)$. But if $s(1,0)-(r, x)$ is in $V_{P}{ }^{+}$we see that either $s>r+P(x)$ or, if $s=r$, that $-x \in V^{+}$and hence $P(x)=0$. In either case $s \geqq r+P(x)$ so we conclude $\bar{U}(r, x)=r+P(x)$. In particular $\bar{U}(B(x))=P(x)$.

2.4 Corollary. Let $f$ be a linear functional defined on a subspace $G$ of an ordered linear space $V$. Suppose that $P$ is a functional defined on $V$, satisfying $(\mathrm{P} 1)-(\mathrm{P} 4)$, and such that $f(x) \leqq P(x)$ for all $x$ in G. Then $f$ is monotonic and there exists a monotonic extension $f^{\prime}$, of $f$, defined on $V$ and such that $f^{\prime}(x) \leqq P(x)$ for all $x$ in $V$.

Proof. If $x \geqq 0$ in $G$ then $f(-x) \leqq P(-x) \leqq P(0)=0$ so we see that $f$ is monotonic. Now consider $V$ as a subspace of the order unit space $\left(V_{P}, u\right)$ of the theorem. Then $f(u)=\sup \{f(x): u \geqq x \in$ $G\} \leqq \sup \{f(x): x \in G, \bar{U}(x) \leqq 1\}=\sup \{f(x): x \in G, P(x) \leqq 1\} \leqq 1$. If we define $f_{1}$ as in 1.1 on $V+R u$ by $f_{1}(v+r u)=f(v)+r f(u)$ then we can extend $f_{1}$ to a monotonic linear functional defined on all of $V_{P}$. If we let $f^{\prime}$ be the restriction of this functional to $V$ we have $f^{\prime}(x) \leqq i n f\left\{f_{1}(r u): x \leqq r u\right\}=\underline{f}(u) \bar{U}(x) \leqq P(x)$ for all $x$ in $V$. 
Note that if $V^{+}=\{0\}$ above then the monotonicity requirements are automatically satisfied and the corollary is just one version of the Hahn-Banach theorem.

Since we can represent the space $\left(V_{P}, u\right)$ as a space of functions on the set $E\left(V_{P}, u\right)$ it follows that $V$ can also be so represented. However this representation may lack one desirable feature; the functions in $E\left(V_{P}, u\right)$ need not be extremal when restricted to $V$. If they were known to be extremal then (as we shall shortly show) we could be sure that finite sups and infs which may exist in $V$ would be preserved as function sups and infs in the function space. In the following theorem we investigate a sufficient condition for the restrictions to be extremal.

2.5 Theonem. Let the hypotheses be those of 2.3. The mapping $B$ has the property that $\{x \in V: B(x)<u\}$ is upward directed if and only if $P$ satisfies the following property:

(P5) $\max \{P(x), P(y)\}=\inf \{P(z): z \geqq x, y\}$,

for all $x$ and $y$ in $V$.

Proof. Suppose that $P$ satisfies (P5) and suppose that $x$ and $y$ are elements of $V$ such that $B(x), B(y)<u$. Then $\max \{P(x), P(y)\}=$ $\max \{\bar{U}(B(x)), \bar{U}(B(y))\}<1$ so there is $z \in V$ such that $x \leqq z, y \leqq z$, and $P(z)=\bar{U}(B(z))<1$ which means $B(z)<u$.

To prove the converse suppose that $P(x)=\max \{P(x), P(y)\}$. For any $\epsilon>0$ if we set $r=(1 /[P(x)+\epsilon])$ we have $\bar{U}(B(r x))=P(r x)<1$. Hence $B(r x)<u$ and $B(r y)<u$ so we can choose $z$ in $V$ with $z \geqq r x$ and $z \geqq r y$ such that $B(z)<u$. But then $P((1 / r) z)=(1 / r) \bar{U}(B(z)) \leqq$ $P(x)+\epsilon$.

Now given $V$ and a functional $P$ on $V$ satisfying (P1)-(P5) we can consider that subset of $E\left(V_{P}, u\right)$ composed of functionals $f$ such that $f(u)=\left(\left.f\right|_{V}\right)(u)$. By theorem 1.1(c) these functionals remain extremal when restricted to $V$. (Note we are identifying $V$ with $B(V)$ here.) If $x$ in $V$ is such that $\bar{U}(x)=P(x)>0$ and $f$ in $E\left(V_{P}, u\right)$ is such that $f(x)=P(x)$ it is easy to see that $f$ is one of these functionals. Consequently if we define for each $x$ in $V$ a function $\tilde{x}$ on this subset of $E\left(V_{P}, u\right)$ by $\tilde{x}(f)=f(x)$ we have a representation which is one-to-one if, and only if, $P$ is such that $x \neq 0$ in $V$ implies $\max \{P(x), P(-x)\}>0$. Also the representation has the property that $\tilde{x} \geqq 0$ implies $x \geqq 0$ if and only if $P$ is such that $P(-x)=0$ implies $x \geqq 0$.

Now we wish to apply the representation results to linear lattices. A linear lattice $V$ is an antisymmetric ordered linear space such that for every pair $\{x, y\}$ in $V$ the least upper bound (written $x \vee y$ ) exists 
in $V$. It is easy to see that in this case the greatest lower bound (written $x \wedge y$ ) also exists in $V$. If we define $x^{+}=x \vee 0, x^{-}=$ $(-x) \vee 0$ and $|x|=x \vee(-x)$ it is not hard to show that $x^{+} \wedge x^{-}=0$, $x=x^{+}-x^{-}$, and $|x|=x^{+}+x^{-}$. The essential tool in the representation of linear lattices is the following characterization of extremal linear functionals due to Hayes.

2.6 Theonem. Let $f$ be a monotonic linear functional defined on an ordered linear space $V$. Then $f$ is extremal if and only if $V=V^{+}-V^{+}$and for each pair $\{x, y\}$ in $V$ we have $\max \{f(x), f(y)\}$ $=\inf \{f(z): x, y \leqq z\}$.

Proof. Let $f$ be extremal on $V$. If $V$ were not equal to $V^{+}-V^{+}$ we could choose a nonzero subspace $H$ complementary to $V^{+}-V^{+}$. If $f$ is zero on $H$ let $g$ be any nonzero linear functional on $H$. Otherwise let $g(y)=2 f(y)$ for all $y$ in $H$. Then for any $x=y+z$ in $H+\left(V^{+}-V^{+}\right)$we define $h(x)=g(y)+f(z)$. Now $0 \leqq h \leqq f$ but $h$ is not equal to $r f$ for any $r$ between 0 and 1 so we have a contradiction.

To show that $\max \{f(x), f(y)\}=\inf \{f(z): x, y \leqq y\}$ it is enough to show that $\max \{f(z): x, 0 \leqq z\}$ for all $x$ in $V$ since $\{z: x, y \leqq z\}=\{z:(x-y), 0 \leqq z\}+y$. Since we have shown $V=V^{+}-V^{+}$we can define a functional $P$ on all of $V$ by $P(x)$ $=\inf \{f(y): x, 0 \leqq y\}$. It is not hard to verify that $P$ satisfies $(P 1)-$ (P4). Suppose $a \in V$ were such that $\max \{f(a), 0\}<P(a)$. Corrollary 2.4 applied to the functional defined on $R a$ by $r a \rightarrow r P(a)$ shows that there exists a linear functional $g$ defined on $V$ such that $g(a)=P(a)$ and $g(x) \leqq P(x)$ for all $x$ in $V$. But if $x$ is in $V^{+}$we have $g(-x) \leqq P(-x)=0$ and $g(x) \leqq P(x)=f(x)$. Hence $g=r f$ for some $r$ between 0 and 1. But this leads to the contradiction $r f(a)=$ $g(a)=P(a)>\max \{f(a), 0\}$.

For the converse let $g$ be a linear functional such that $0 \leqq g \leqq f$. If $f$ is zero then $g$ is zero since $V=V^{+}-V^{+}$. Otherwise let $a$ be an element of $V^{+}$such that $f(a)>0$. Then for any $x$ in $V$ we have, setting $y=x-(f(x) / f(a)) a$;

$$
\begin{aligned}
0 & =f(y)=\max \{f(y), 0\}=\inf \{f(z): y, 0 \leqq z\} \\
& \geqq \inf \{g(z): y, 0 \leqq z\} \geqq \max \{g(y), 0\} .
\end{aligned}
$$

Hence $g(x) \leqq(f(x) / f(a)) g(a)$ and since the same holds for $(-x)$ we conclude that $g=r f$ where $r=g(a) / f(a)$. Since $0 \leqq g \leqq f$ it is clear that $0 \leqq r \leqq 1$. 
2.7 Conollary. Let $V$ be a linear lattice and let $f$ be a monotonic linear functional defined on $V$. Then $f$ is extremal if and only if $f(x \vee y)=\max \{f(x), f(y)\}$ for all $x$ and $y$ in $V$.

Thus when an order unit space $(V, u)$ is also a linear lattice the mapping of $V$ into $\hat{V}$ is such that sups in $V$ become pointwise function sups in $\hat{V}$. Furthermore in this case we have a considerable amount of information about the structure of $E(V, u)$ and $\hat{V}$ as we see from the following theorem of Kakutani.

2.8 TheоReм. Let $V$ be a linear lattice with order unit $u$ and let $E(V, u)$ be given the weak topology by the functions in $\hat{V}$. Then $E(V, u)$ is compact and $\hat{V}$ is dense in the space of continuous functions on $E(V, u)$.

Proof. The set $E(V, u)$ is contained in the product space $\prod_{x \in V}[\underline{U}(x), \bar{U}(x)]$ and this set is compact in the product topology by Tychonoff's theorem. If we consider the functions $\hat{x}$, for $x$ in $V$, defined on the product space by $\hat{x}(f)=f(x)$ it is clear that the weak topology by these functions is the same as the product topology. For any $x$ and $y$ in $V$ the functions $f \rightarrow f(x \vee y)$ and $f \rightarrow \max \{f(x), f(y)\}$ are continuous and hence the set where they coincide is closed. Intersecting over all $x$ and $y$ in $V$ we find that the sup-preserving mappings in the product space form a closed subspace. Using the same technique for linearity we find that the subset of extremal linear functionals is closed in the product space. If we intersect this set with the closed set $\hat{u}^{-1}(1)$ we get $E(V, u)$ which is hence a compact set.

Since $\hat{u}$ is the constant one function on $E(V, u)$ it is clear that $\hat{V}$ is a function lattice subspace of the continuous functions on $E(V, u)$ which contains constants and separates points. It follows from the Stone-Weierstrass theorem that $\hat{V}$ is dense in the space of continuous functions.

3. Representation of Linear Lattices Without Unit. If a linear lattice lacks a unit it is still possible to represent it but it is necessary to use extended-real-valued functions. First we need a preliminary result.

3.1 Lemma. Let $f$ be a maximal extremal functional defined on a subspace $G$ of a linear lattice $V$ (i.e., $f \in E(V)$ ). Then $G$ is a sublattice of $V$ and, for every $x$ in $V$ such that there exists $y$ in $G$ with $y \leqq x^{+}$and $f(y)>0$, we have $f\left(x^{-}\right)=0$.

Proof. From theorem 1.2 it is clear that if $x$ and $y$ are in $G$ then $x \vee y$ and $x \wedge y$ are in $G$ since $G=G^{+}-G^{+}$by theorem 2.6. If 
$x$ in $V$ and $y$ in $G$ are such that $y \leqq x^{+}$and $f(y)>0$ let $z$ be any element of $G$ which is smaller than $x^{-}$. We have $z \wedge y \leqq x^{-} \wedge x^{+}$ $=0$ and hence $0 \geqq f(z \wedge y)=\min \{f(z), f(y)\}$. Since $f(y)>0$ we conclude that $f(z) \leqq 0$. Thus $\underline{f}\left(x^{-}\right)=0$.

Now given a maximal extremal functional $f$ we first extend the definition of $f$ to all of $G+V^{+}$by allowing it to assume the value $+\infty$. We define an extended-real-valued function $F$ on $V$ by: $F(x)=\underline{f}\left(x^{+}\right)-\underline{f}\left(x^{-}\right)$for all $x$ in $V$. This is well-defined since if $\underline{f}\left(x^{+}\right)=+\infty$ the lemma shows that $\underline{f}\left(x^{-}\right)=0$. In addition if $x$ is in $\operatorname{dmn} f$ then $x^{+}$and $x^{-}$are also in $\operatorname{dmn} f$ by the lemma and hence $F(x)=f(x)$. Finally if $x$ is not in $\operatorname{dmn} f$ then we must have either $\underline{f}\left(x^{+}\right)=+\infty$ or $\underline{f}\left(x^{-}\right)=+\infty$ by theorem 1.1 and maximality of $f$. We conclude that $F$ is finite only on $\operatorname{dmn} f$ and coincides with $f$ there.

3.2 Theorem. Let $V$ be a linear lattice and let $f \in E(V)$. If we define $F$ on $V$ by $F(x)=\underline{f}\left(x^{+}\right)-\underline{f}\left(x^{-}\right)$for each $x$ in $V$ then we have, for any $x, y$ in $V$ and $r$ in $R$ :

(1) $F(r x)=r F(x)$ (where $0( \pm \infty)$ is defined to be zero.)

(2) $F(x+y)=F(x)+F(y)$ (whenever the right side makes sense.)

(3) $F(x \vee y)=\max \{F(x), F(y)\}$.

Proof. Number (1) is a routine calculation. In number (2) we note first that additivity is trivial when $x$ and $y$ are in $\operatorname{dmn} f$. Suppose now that $F(x)>-\infty$ and $F(y)=+\infty$. Then since $(x+y)^{+}+x^{-}+$ $y^{-}=x^{+}+y^{+}+(x+y)^{-} \geqq y^{+}$we have $\underline{f}\left((x+y)^{+}\right)+f\left(x^{-}\right)+$ $f\left(y^{-}\right)=\underline{\underline{f}}\left((x+y)^{+}+x^{-}+y^{-}\right) \geqq \underline{f}\left(y^{+}\right)=+\infty$. Thus $F(x+y)=$ $+\infty=\overline{\bar{F}}(x)+F(y)$. Finally if $F(x)<+\infty$ and $F(x)<+\infty$ and $F(y)$ $=-\infty$ then $F(x+y)=-F(-x-y)=-\infty=F(x)+F(y)$.

As for result (3) it is clear that if $x$ and $y$ are in $\operatorname{dmn} f$ then it follows from corollary 2.7. Suppose $F(y)=+\infty$. Then $(x \vee y)^{+}=$ $(x \vee y) \vee 0=x \vee y^{+} \geqq y^{+}$so $\underline{f}\left((x \vee y)^{+}\right)=+\infty$ and we have $\max \{F(x), F(y)\}=+\infty=F(x \vee y)$. If $F(y)=-\infty$ and $x$ is in $\operatorname{dmn} f$ then $x \leqq x \vee y \leqq x \vee y^{+} \in \operatorname{dmn} f$ implies $x \vee y \in \operatorname{dmn} f$. Choose $z$ in $\operatorname{dmn} f$ such that $z \leqq y^{-}$and $|f(x)| \leqq f(z)$. Then $x \leqq x \vee y \leqq x \vee\left(y^{+}-z\right)$ implies $f(x) \leqq f(x \vee y) \leqq f\left(x \vee\left(y^{+}-z\right)\right)$ $=\max \{F(x), \quad-f(z)\}=f(x)$. Thus $F(x \vee y)=f(x \vee y)=f(x)=$ $\max \{\boldsymbol{F}(\boldsymbol{x}), \boldsymbol{F}(\boldsymbol{y})\}$. Finally if $F(x)=-\infty=F(y)$ then for any positive integer $n$ there are $a$ and $b$ indmn $f$ such that $a \leqq x^{-}, b \leqq y^{-}$and $f(a \wedge b)$ $=\min \{f(a), f(b)\}>n$. Then $a \wedge b \leqq x^{-} \wedge y^{-}=(x \vee y)^{-}$implies $\underline{f}\left((x \wedge y)^{-}\right)>n$. Hence $F(x \wedge y)=-\infty=\max \{F(x), F(y)\}$.

$=-\infty=\max \{F(x), F(y)\}$. 
In order to get a representation of a linear lattice $V$ we first note that Zorn's lemma allows us to assert the existence of a family $Q$ of nonzero elements of $V^{+}$which is maximal with respect to the property of being disjoint (i.e., $x \neq y$ in $Q$ implies $x \wedge y=0$.) Given such a set $Q$ we define $E(V, Q)$ to be the set of those $f$ in $E(V)$ such that $f(q)=1$ for some $q$ in $Q$. Given $x$ in $V$ we define an extended-realvalued function $\hat{x}$ on $E(V, Q)$ by $\hat{x}(f)=F(x)$. By the previous theorem this embeds $V$ as a subset $\hat{V}$ of the set of extended-realvalued functions on $E(V, Q)$ in such a way that linear and lattice operations are preserved as pointwise operations when they make sense.

3.3 Theorem. Let $V$ be a linear lattice, let $Q$ be a maximal disjoint set and suppose that $E(V, Q)$ is given the weak topology determined by the functions in $\hat{V}$. Then $V$ is Archimedean (note Archimedean is equivalent to almost Archimedean in a linear lattice) if and only if the representation is one-to-one and the functions in $\hat{V}$ are continuous and almost finite (i.e., finite on a dense subset of $E(V, Q))$.

Proof. It is clear that the functions in $\hat{V}$ are continuous in the weak topology. Suppose that the mapping $x \rightarrow \hat{x}$ is one-to-one and that the functions in $\hat{V}$ are almost finite. Then if $\{x, y\}$ is a pair in $V$ such that $n x \leqq y$ for all $n=1,2, \cdots$ we have $n x^{+} \leqq y^{+}$implies $n \hat{x}^{+} \leqq \hat{y}^{+}$for all $n=1,2, \cdots$. Consequently $\hat{x}^{+}(f)=0$ for each $f$ such that $\hat{y}^{+}(f)<+\infty$ and hence $\hat{x}^{+}=0$ since it is a continuous function which is zero on a dense set. Since the mapping is one-to-one we see that $x^{+}=0$ and thus $x=-x^{-} \leqq 0$.

For the converse we first prove a lemma on the weak topology.

3.4 Lemma. The weak topology on $E(V, Q)$ determined by the functions in $\hat{V}$ is the same as the weak topology determined by the functions in the subset $\bigcup_{q \in Q}\left(R q-V^{+}\right) \cap\left(R q+V^{+}\right)$.

Proof. We need only show that every basic neighborhood in the first topology contains a basic neighborhood in the second. Given $f \in E(V, Q)$ let a basic neighborhood of $f$ be determined by $\left\{y_{1}, \cdots, y_{n}\right\} \subset V$ and $\left\{a_{1}, \cdots, a_{n}\right\} \subset R$ in the following way: $f \in N=\bigcap_{i=1}^{n}\left\{g \in E(V, Q): \hat{y}_{i}(g)>a_{i}\right\}$. (Note that this does describe all basic neighborhoods since $\left\{g \in E(V, Q): b<\hat{x}(g)<b^{\prime}\right\}$ $=\{g \in E(V, Q): \hat{x}(g)>b\} \cap\left\{g \in E(V, Q):(-\hat{x})(g)>-b^{\prime}\right\}$.) Choose $q$ in $Q$ such that $f(q)=1$ and define $z=\left(q \wedge\left(y_{1}-a_{1} q\right) \wedge\right.$ $\left.\cdots \wedge\left(y_{n}-a_{n} q\right)\right)^{+}$. Then $N \supset\{g \in E(V, Q): \hat{z}(g)>0\}$ contains $f$ and $z \in\left(R q-V^{+}\right) \cap\left(R q+V^{+}\right)$. 
Now suppose that $V$ is Archimedean. If $x \neq y$ in $V$ then either $(y-x)^{+}$or $(y-x)^{-}$is nonzero. Suppose $(y-x)^{+} \neq 0$. Then, by maximality, there exists $q \in Q$ such that $(y-x)^{+} \wedge q>0$. Let $p=$ $|x|+|y|+q$ and let $V_{p}=\left(R p-V^{+}\right) \cap\left(R p+V^{+}\right)$. On the order unit space $V_{p}$ there exists $f \in E\left(V_{p}, p\right)$ such that $f\left((y-x)^{+} \wedge q\right)$ $\neq 0$ by theorem 2.2. If we extend $(1 / f(q)) f$ to an element $f^{\prime}$ in $E(V, Q)$ we find $f^{\prime}(x) \neq f^{\prime}(y)$ so the mapping is one-to-one.

Finally suppose that there is $x$ in $V$ such that the set $T=$ $\{f \in E(V, Q): \hat{x}(f)= \pm \infty\}$ has non-empty interior. By the lemma there exists $q$ in $Q$ and $z \in\left(R q-V^{+}\right) \cap\left(R q+V^{+}\right)$such that $T \supset N=\{f \in E(V, Q): \hat{z}(f)>0\} \neq \varnothing$. But then $n \hat{z}(f) \leqq|\hat{x}|(f)$ for all $f \in E(V, Q)$ and all $n=1,2, \cdots$ implies that $n z \leqq|x|$ for all $n=1,2, \cdots$ just as in the previous paragraph and we conclude that $z \leqq 0$. This contradicts $N \neq \varnothing$.

We now determine a necessary and sufficient condition that $E(V, Q)$ be compact and investigate when, in this case, the representing functions are real-valued rather than extended-real-valued.

3.5 Theоnem. Let $V$ be a linear lattice, let $Q$ be a maximal disjoint set and suppose that $E(V, Q)$ is given the weak topology determined by the functions in $\hat{V}$. Then $E(V, Q)$ is compact if and only if $Q$ is finite. If, in addition, $V$ is Archimedean, then the functions in $\hat{V}$ take only finite values if and only if $u=\sum\{q: q \in Q\}$ is an order unit for $V$.

Proof. Let $T_{q}=\{f \in E(V, Q): \hat{q}(f)>0\}$, an open set in the weak topology. For any $p \neq q$ in $Q$ and any $f \in E(V, Q)$ we have $\min \{f(p), f(q)\}=f(p \wedge q)=f(0)=0$ so we see that $T_{q} \cap T_{p}=\varnothing$ and $T_{q}=\{f \in E(V, Q): \hat{q}(f)=1\}$, a closed set in the weak topology. Clearly if $Q$ is infinite then $E(V, Q)$ is an infinite disjoint union of the family $\left\{T_{q}: q \in Q\right\}$ of open sets and hence is not compact. If $Q$ is finite and we let $V_{q}=\left(R q-V^{+}\right) \cap\left(R q+V^{+}\right)$ for each $q$ in $Q$ then it is easy to see from Lemma 3.4 that the mapping $\left.f \rightarrow f\right|_{V_{q}}$ maps $T_{q}$ homeomorphically onto $E\left(V_{q}, q\right)$. Since this last set is compact by Theorem 2.8 we see that $E(V, Q)$ is compact as a finite union of compact sets.

If we have, further, that $V$ is Archimedean, then suppose that the functions in $\hat{V}$ take only finite values. Given any $x \in V$, by compactness of $E(V, Q)$, there exists a positive integer $n$ such that $-n \leqq \hat{x} \leqq n$. Since the constant function one is the representation of $u$ we conclude by theorem 3.3 that $(n u-|x|)^{-}=0$ or $-n u \leqq x \leqq n u$ as desired. The converse follows from the fact that $-n u \leqq x \leqq n u$ implies $-n \leqq \hat{x} \leqq n$. 
4. Applications to the Theory of the Choquet Boundary. The representation of an ordered linear space $V$ as a space of functions on the set of those monotonic linear functionals which happen to be extremal has the advantage that the lattice structure, if any, is preserved. We will now show that this representation gives other interesting results when $V$ is given in advance as a subspace of $C(X)$, for $X$ a compact Hausdorff space.

4.1 Theorem. Let $X$ be a compact Hausdorff space and let $V$ be a subspace of $C(X)$, the continuous real-valued functions on $X$, which contains the constant functions. If we define a mapping $A$ from $X$ to the space of monotonic linear functionals on $V$ by $A(s)(x)=x(s)$ for all $s$ in $X$ and $x$ in $V$ then the range of the mapping $A$ contains $E(V, 1)$.

Proof. Given $x \in V$ let $S(x)=\{t \in X: x(t)>0\}^{-}$. By induction we can extend theorem 2.6 to show that for each finite subset $\left\{x_{1}, \cdots, x_{n}\right\} \subset V$ and each $f \in E(V, 1)$ we have $\min \left\{f\left(x_{1}\right), \cdots\right.$, $\left.f\left(x_{n}\right)\right\}=\sup \left\{f(z): z \leqq x_{i}\right.$ for all $\left.i=1, \cdots, n\right\}$. From this fact we see easily that, for a fixed $f$ in $E(V, 1)$, the family $\{S(x): f(x)>0\}$ has the finite intersection property. By compactness we can choose a point $t$ in the intersection of this family. For any $x$ in $V$ and $\epsilon>0$, we have $f(x-(f(x)-\epsilon) 1)>0$ so we conclude that $t \in$ $S(x-(f(x)-\epsilon) 1)$. Continuity then shows that $x(t) \geqq f(x)-\epsilon$. Since $\epsilon$ was arbitrary we see that $x(t) \geqq f(x)$. The same calculation for $(-x)$ shows that $x(t)=f(x)$ so we have $f=A(t)$ as desired.

We define a boundary for the subspace $V$ of $C(X)$ to be any subset $B$ of $X$ such that every function in $V$ achieves its maximum on $B$. It is easy to see that, in this case, $V$ is isomorphic with $\left.V\right|_{B}$; i.e., we can ignore the "superfluous points" in $X \backslash B$. It would clearly be useful to take the smallest boundary if such a set existed. The next theorem shows that a smallest closed boundary (called the Shilov boundary) always exists.

4.2 Theorem. Let $X$ be a compact Hausdorff space and let $V$ be a subspace of $C(X)$ which contains the constant functions. Then the set $A^{-1}(E(V, 1))$ is a boundary for $V$ and is contained in every closed boundary for $V$.

Proof. If we designate the constant function 1 by $u$ then it is clear that, for any $x$ in $V, \bar{U}(x)=\max \{x(t): t \in X\}$. By theorem 2.1 there exists $f$ in $E(V, u)$ such that $f(x)=\bar{U}(x)$ so we see that $x$ achieves its maximum on any element of $A^{-1}(f)$. 
If $Y \subset X$ is any closed boundary for $V$ consider the restriction mapping $\left.x \rightarrow x\right|_{Y}$ from $V$ into $R^{Y}$. If $\left.x\right|_{Y} \geqq 0$ then the fact that $Y$ is a boundary shows that $x \geqq 0$ so we conclude that the mapping is an isomorphism. Let the mapping $A_{1}$, from $Y$ into the space of monotonic linear functionals on $\left.V\right|_{Y}$, be defined by $A_{1}(s)\left(\left.x\right|_{Y}\right)=\left(\left.x\right|_{Y}\right)(s)$ for all $s$ in $Y$ and $x$ in $V$. Since $V$ is isomorphic with $\left.V\right|_{Y}$ we can identify $E(V, 1)$ with $E\left(\left.V\right|_{Y},\left.1\right|_{Y}\right)$ and, since $Y$ is compact, theorem 4.1 shows that $E(V, 1)$ is contained in the range of $A_{1}$. Hence $Y \supset A_{1}^{-1}(E(V, 1))$ $=A^{-1}(E(V, 1))$.

The subspace $V$ of $C(X)$ is said to separate points of $X$ if, for each pair $s \neq t$ in $X$ there exists $x$ in $V$ with $x(s) \neq x(t)$.

4.3 Theorem. Let $X, V$, and $A$ be as in theorem 5.1 and suppose that $V$ separates points of $X$. An element $s$ of $X$ is in $A^{-1}(E(V, 1))$ if and only if $\mathrm{A}(\mathrm{s})$ has a unique monotonic linear extension from the subspace $\mathrm{V}$ to $\mathrm{C}(\mathrm{X})$.

Proof. Suppose $s$ is in $A^{-1}(E(V, 1))$. Let $W$ be the set of finite infs from $V$. Since $W$ is a wedge it is easy to see that $W-W$ is a subspace of $C(X)$ and since, for $x$ and $y$ in $W$, we have $(x-y) \wedge 0$ $=(x \wedge y)-y \in W-W$ it is not hard to show that $W-W$ is a sublattice of $C(X)$. If $z=x_{1} \wedge \cdots \wedge x_{n}$ is any element of $W$ then $\underline{A(s)}(z) \leqq \overline{A(s})(z) \leqq \min \left\{A(s)\left(x_{1}\right), \cdots, A(s)\left(x_{n}\right)\right\}=\underline{A(s)}(z)$ where the last equality follows from the inductive extension of theorem 2.6. Since every monotonic extension of $A(s)$ is trapped between $\underline{A(s)}$ and $\overline{A(s)}$ we conclude that $A(s)$ has a unique monotonic extension to $W$ and hence to $W-W$. But this subspace is dense in $C(X)$ by the StoneWeierstrass theorem so, if $f$ is any element of $C(X)$ and $\epsilon$ is any positive number, there exists $x$ in $W-W$ such that $x-\epsilon \leqq f \leqq x+\epsilon$. This shows that any monotonic linear function on $W-W$ has a unique monotonic extension to $C(X)$.

Conversely suppose that $A(s)$ has a unique monotonic extension to $C(X)$. Then, for any $x$ and $y$ in $V$, theorem 1.1 and the remark following show that we must have $\underline{A}(s)(x \vee y)=\bar{A}(s)(x \vee y)$; otherwise we could get two different monotonic extensions to $V+R(x \vee y)$ and extend these to different extensions on $C(X)$. But this gives $\inf \{A(s)(z): x, y \leqq z\}=\overline{A(s)}(x \vee y)=\underline{A(s)}(x \vee y) \leqq \max \{A(s)(x)$, $A(s)(y)\}$ where the last inequality follows from: $z \leqq x \vee y$ implies $A(s)(z)=z(s) \leqq(x \vee y)(s)=\max \{x(s), y(s)\}=\max \{A(s)(x), A(s)(y)\}$. We conclude that the condition in theorem 2.6 is satisfied so $A(s)$ is extremal.

The set $A^{-1}(E(V, 1))$ is called the Choquet boundary for $V$. Theorem 
5.2 gives the two results due to Bauer [1] that the Choquet boundary really is a boundary and that its closure is the Shilov boundary. Theorem 5.3 gives an equivalent definition which is very useful in the measure-theoretic approach to Choquet boundaries.

We now show how the Krein-Milman theorem follows from the previous results. A subset $X$ of a linear space $L$ is said to be convex if, for every $s, t$ in $X$ and $0 \leqq r \leqq 1$ in $R$, the element $r s+(1-r) t$ is also in $X$. A point $t$ of $X$ is said to be an extreme point if $t=r s_{1}+$ $(1-r) s_{2}$ for $0 \leqq r \leqq 1$ and $s_{1} \neq s_{2}$ in $X$ implies $r=0$ or $r=1$. To aid the reader's geometric intuition we note that the extreme points of a circular disc are those points on the circumference while the extreme points of a square are the four vertices.

4.4 Theorem. Let $X$ be a convex set in a linear space L. Suppose there is a topology on $X$ such that $X$ is compact and the space of linear functionals on $L$ whose restrictions to $X$ are continuous on $X$ in this topology separates points of $X$. Then $X$ has an extreme point.

Proof. Let $H$ be the space of functions on $X$ which are continuous on $X$ and which are the restrictions of linear functionals on $L$ and let $V=H+R 1$. Since $X$ is compact the Choquet boundary with respect to $V$ is nonempty. We will show that every Choquet boundary point is an extreme point. Suppose $t \in X$ is not an extreme point. Then $t=$ $r s_{1}+(1-r) s_{2}$ for $0<r<1$ and $s_{1} \neq s_{2}$ in $X$. Then for any $f \geqq 0$ in $V$ we have $0 \leqq r A\left(s_{1}\right)(f) \leqq r A\left(s_{1}\right)\left(f\left(+(1-r) A\left(s_{2}\right)(f)\right.\right.$ $=r f\left(s_{1}\right)+(1-r) f\left(s_{2}\right)=f\left(r s_{1}+(1-r) s_{2}\right)=f(t)=A(t)(f)$. Thus $0 \leqq r A\left(s_{1}\right) \leqq A(t)$ while $r A\left(s_{1}\right)$ is not a multiple of $A(t)$ since there is a function in $V$ which is zero on $t$ and nonzero on $s_{1}$. Thus $t$ is not in the Choquet boundary.

By the use of measure-theoretic techniques it can be shown [1] that the set of extreme points is equal to the Choquet boundary.

5. Limitations of the Representation Theory. No presentation of a theory is complete without some discussion of its weaknesses as well as its strengths. The most useful result is theorem 2.8 of Kakutani which allows us to conclude, with an extra completeness assumption on $V$, that $V$ is isomorphic to $C(E(V, u))$. However the two assumptions that $V$ is a linear lattice and has an order unit are essential here. If we drop the requirement that $V$ be a linear lattice then $E(V, u)$ is no longer compact in general and $V$ is not necessarily dense in $C(E(V, u))$. If we retain the lattice requirement and drop the order unit then we must utilize extended-real-valued functions. If we replace the order unit restriction by the assumption that a functional 
$P$, satisfying (P1)-(P5) of theorems 2.3 and 2.5, is defined on $V$ then we can represent $V,[4]$, as a function sublattice of $C(X)$ where $X$ is a compact Hausdorff space but it is no longer dense in general. In fact, if we assume that the function $x \rightarrow \max \{P(x), P(-x)\}$ is a complete norm on $V$ we find that the representing space is an intersection of spaces of the form $H_{a}$, where each $H_{a}$ is defined in terms of a pair $t_{a}, t_{a}{ }^{\prime}$ in $X$ and a real number $r_{a}, 0 \leqq r_{a} \leqq 1$ in the following way:

$$
H_{a}=\left\{f \in C(X): f\left(t_{a}\right)=r_{a} f\left(t_{a}{ }^{\prime}\right)\right\} .
$$

The necessity of utilizing extended-real-valued functions in the absence of an order unit (it really is a necessity in general if we wish to preserve the lattice structure. See [7]) leads to problems with addition. Because of the ambiguity involved in adding $+\infty$ and $-\infty$ the set of almost finite continuous functions on $X$, even when $X$ is compact, is not necessarily a linear space. However if the topology of $X$ is such that $X$ is a Stone space (i.e., the closure of every open set is open) or even a basically disconnected space (i.e., the closure of every open $F_{\sigma}$ set is open) then with each pair $\{f, g\}$ of almost finite continuous functions we can associate uniquely a new almost finite continuous function $h$ such that $h(t)=f(t)+g(t)$ whenever the right side is defined. If we define " $\oplus$ " by $f \oplus g=h$ then the set of almost finite continuous functions becomes a linear space. If $V$ is such that upper bounded families have a least upper bound (resp. countable upper bounded families have a least upper bound) then $E(V, Q)$ is a Stone space (resp. $E(V, Q)$ is basically disconnected) and $V$ is a subspace of the linear space of almost finite continuous functions.

One virtue of the representation for lattices is that we can use it to establish finite lattice equalities and inequalities such as ||$x|-| y||$ $\leqq|x-y|$ merely by thinking of the elements as extended-real-valued functions and checking that the result holds pointwise. In short, if it is true for the real numbers, it is true for a linear lattice, [10]. However lattice results involving infinite sups or infs cannot be reduced to the same results in the real numbers since infinite sups or infs in $\hat{V}$ need not be pointwise sups or infs, [8].

Kakutani has shown that, in the situation of theorem 2.6, $E(V, u)$ is unique up to homeomorphism and has applied his result to show the existence and uniqueness of the Stone-Cech compactification. However in the general case uniqueness does not hold. Even if we have two one-element maximal disjoint sets $Q_{1}$ and $Q_{2}$ it is not true in general that $E\left(V, Q_{1}\right)$ is homeomorphic to $E\left(V, Q_{2}\right)$. Bernau, [2], has a representation theory in which the domain of the representing functions is unique up to homeomorphism but it has the disadvantage that 
if we start with $V=C(X)$ for $X$ a compact Hausdorff space the domain of the functions in $\hat{V}$ need not be homeomorphic to $X$. The fact that this does happen in the theory presented here is an easy consequence of theorems 2.6 and 4.1 .

A final weakness is the fact that the theory says nothing about the representation when $V$ is not a lattice and lacks an order unit. If $V$ is antisymmetric and Archimedean we can use a completion process, [7], [9] to embed $V$ in a linear lattice and proceed to represent the containing linear lattice. This process however has the same disadvantage cited for Bernau's theory in the previous paragraph.

Finally, no discussion of extremal linear functionals would be complete without a mention of their application to partially ordered linear algebras. If $(V, u)$ is given a multiplication such that the product of positive elements is positive and such that $u$ is the multiplicative identity then the functionals in $E(V, u)$ are just the nonzero monotonic multiplicative linear functionals. For details of this theory the reader may consult [4] .

\section{REFERENCES}

1. H. Bauer, Schilowscher Rand und Dirichletsches Problem. Ann. Inst. Fourier 11, 89-136 (1961).

2. S. J. Bernau, Unique Representation of Archimedean Lattice Groups and Normal Archimedean Lattice Rings, Proc. London Math. Soc. (3) 15 (1965), 599-631.

3. G. Jameson, Ordered Linear Spaces, Springer-Verlag, Berlin 1970.

4. R. V. Kadison, A Representation Theory for Commutative Topological Algebras, Mem. Amer. Math. Soc. 7 (1951).

5. S. Kakutani, Concrete Representations of Abstract (M)-spaces, Ann. of Math. 42, 994-1024 (1941).

6. W. A. J. Luxembourg and A. C. Zaanen, Riesz Spaces I, North Holland, Amsterdam, 1971.

7. H. Nakano, Modern Spectral Theory, Maruzen, Tokyo, 1950.

8. D. Papert, A Representation Theory for Lattice Groups, Proc. London Math. Soc. (3) 12 (1962), 100-120.

9. A. Peressini, Ordered Topological Vector Spaces, Harper and Row, New York (1967).

10. B. Z. Vulikh, Introduction to the Theory of Partially Ordered Spaces. Wolters-Noordhoff, Groningen (1967).

11. K. Yosida, On Vector Lattice with a Unit, Proc. Imp. Acad. Tokyo 17 (1940-41), 121-124.

University of New Mexico, Albuquerque, New Mexico 87131 
